\title{
Glucocorticoid and mineralocorticoid receptor activation modulates postnatal growth
}

\author{
Erin Faught and Mathilakath M Vijayan \\ Department of Biological Sciences, University of Calgary, Calgary, Alberta, Canada \\ Correspondence should be addressed to M M Vijayan: matt.vijayan@ucalgary.ca
}

\begin{abstract}
During early development, stress or exogenous glucocorticoid (GC) administration reduces body mass in vertebrates, and this is associated with the glucocorticoid receptor (GR) activation. Although GCs also activate the mineralocorticoid receptor $(M R)$, the physiological significance of MR activation on early developmental growth is unknown. We tested the hypothesis that activation of both GR and MR are required for postnatal growth suppression by GCs. Differential regulation of GR and MR activation was achieved by using ubiquitous GR- (GRKO) and MR- (MRKO) knockout zebrafish (Danio rerio) in combination with exogenous cortisol treatment. MR activation increased protein deposition in zebrafish larvae and also upregulated lepa and downregulated lepr transcript abundance. Cortisol treatment reduced body mass and protein content in the WT, and this corresponded with the upregulation of muscle proteolytic markers, including murf1 and redd 1 by GR activation. The combined activation of MR and GR by cortisol also upregulated the $g h$ and igf 1 transcript abundance, and insulin expression compared to the WT. However, cortisol-mediated reduction in body mass and protein content required the activation of both MR and GR, as activation by GR alone (MRKO + cortisol) did not reduce the larval protein content. Collectively, our results indicate that MR activation favors protein deposition and GR activation stimulates proteolysis, while their combined activation is involved in cortisol-mediated growth suppression. Overall, this work provides insight into the physiological significance of MR activation in regulating protein deposition during early development at a systems level.
\end{abstract}

Key Words
- glucocorticoid receptor
- mineralocorticoid
receptor
- growth
- zebrafish
- skeletal muscle
metabolism

\section{Introduction}

Both intrauterine and early postnatal environments program various physiological systems in vertebrates. Major factors affecting fetal growth include maternal nutrition and fetal stress. Excess glucocorticoids (GCs) are of particular concern as exposure to GCs in utero reduces skeletal muscle mass in rats, and this is independent of maternal nutrition (Gokulakrishnan et al. 2012). The growth of skeletal muscle mass depends on the balance between the anabolic and catabolic processes, which are driven to a large extent by complex endocrine signals (Kuo et al. 2013). Increase in skeletal muscle mass is an important component of postnatal growth because it comprises approximately $40 \%$ of adult body weight in mammals (Shimizu et al. 2011) and $>50 \%$ in fish (Mommsen 2001, Sadoul \& Vijayan 2016). Muscle growth is driven primarily by anabolic hormones, including the growth hormone (GH) /insulin-like growth factor 1 (IGF1) axis, and the associated activation of 
P13K/AKT/mTOR pathway (Shimizu et al. 2011). Muscle loss is associated with enhanced proteolysis, which involves the activation of the ubiquitin pathway, including transcriptional activation of E3 ligase-encoding genes, atrogin-1 and murf1, and the autophagic/lysosomal pathway (Kuo et al. 2013). GCs released in response to stress stimulate proteolysis, and a key target for these steroids is the regulated in development and DNA damage response 1 (REDD1), which reduces skeletal muscle mass by inhibiting mTORC1/S6K pathway (Shimizu et al. 2011, Lipina \& Hundal 2016).

At a systems level, postnatal stress, and the attendant rise in GCs cause dysregulation of normal growth trajectories (Charmandari et al. 2005, Kelly et al. 2012). This negative effect of GCs on growth is mediated by the glucocorticoid receptor (GR) activation of proteolytic pathways (Shimizu et al. 2011, Lipina \& Hundal 2016, Britto et al. 2018). Indeed, both murf1 and redd1 are under the transcriptional control of GR in mammals (Kuo et al. 2013), and also in zebrafish (Danio rerio) (Faught \& Vijayan 2019a). In addition, ubiquitous knockout of GR in zebrafish led to greater body mass, including higher protein and fat, supporting a role for GCs in regulating skeletal muscle mass (Facchinello et al. 2017, Faught \& Vijayan 2019a). In teleosts, cortisol is the primary GC and the mode of action involves the activation of either GR and/or mineralocorticoid receptor (MR). Both these receptors are ligand-bound transcription factors and regulate gene expression by binding to either the glucocorticoid response elements (GREs) or the mineralocorticoid response elements (MREs) in the promoter regions of target genes (Kuo et al. 2013). The primary role of cortisol during stress is to increase glucose availability by increasing gluconeogenesis, as well as promoting muscle proteolysis to channel the C3 substrates for gluconeogenesis in the liver (Mommsen et al. 1999). Consequently, chronic cortisol stimulation leads to glucose intolerance and increased muscle proteolysis, leading to reduced skeletal muscle mass. The role of MR in regulating skeletal muscle mass and protein content is less well defined. Although MRs are present in the mammalian skeletal muscle and responsive to aldosterone (Chadwick et al. 2015), a physiological role for GC-MR activation in skeletal muscle growth is yet to be characterized. A recent study suggested that MR bind to GREs to mediate GR function in mammals (Mifsud \& Reul 2016), and we also recently showed that lipid accumulation in zebrafish larvae requires the activation of both GR and MR, supporting a conserved role for corticosteroid receptors (CRs) interactions in energy metabolism (Faught \& Vijayan 2019b).

In addition to regulation of proteolysis, GCs also exert effects on growth by modulating the functioning of the growth axis in fish (Mommsen 2001, Madison et al. 2015, Sadoul \& Vijayan 2016) and mammals (Yuen et al. 2013). Much of the mechanistic work has been carried out in mammals, and GCs have been shown to either inhibit or stimulate growth hormone $(\mathrm{GH})$ release (Shapiro et al. 1978, Sartin et al. 1994). The use of GCs-based therapeutics during childhood has also been associated with long-term dysregulation of growth, suggesting effects on developmental programming (Charmandari et al. 2005, Kelly et al. 2012). The lack of consensus in GCs-mediated GH regulation has also been reported in teleosts. For instance, chronic stress has been associated with an increase in GH and IGF1 levels in Atlantic salmon parr (Salmo salar) (McCormick et al. 1998) and adult rainbow trout (Oncorhynchus mykiss) (Madison et al. 2015), whereas acute stress either results in no change or a decrease in circulating GH levels (Pickering 1993, Shepherd et al. 2011). Stress and the attendant rise in GCs can also indirectly affect growth by reducing food consumption and GH signaling in fish (Bernier 2006, Ortega et al. 2013). Altogether, GCs effect on muscle growth may involve differential regulation of anabolic and catabolic processes by CRs, but the mechanisms are elusive.

Here, using zebrafish larvae as a model of rapid growth, we tested the hypothesis that GC reduces postnatal growth, and this is mediated by both MR and GR activation. Unlike other teleosts, zebrafish lacks a GR paralog (Alsop \& Vijayan 2008, 2009), making them an ideal model for corticosteroid receptor loss-of-function studies. Also, teleost lacks aldosterone (Baker et al. 2013), and since we have the ubiquitous GR- (GRKO) and MR- (MRKO) knockout zebrafish strains (Faught \& Vijayan 2018a, 2019a), this allowed us to test the differential role of these two receptors in mediating the GC effect on postnatal growth in zebrafish larvae. Our results indicate that MR activation has an anabolic effect and favors protein deposition during postnatal growth, while GR activation stimulates proteolysis, leading to the suppression of growth in response to cortisol stimulation. Interestingly, cortisol-mediated growth suppression was observed only in the presence of both receptors, because in the absence of MR activation, GR alone did not cause a reduction in protein content or growth in zebrafish larvae.
(C) 2020 Society for Endocrinology Published by Bioscientifica Ltd. Printed in Great Britain 


\section{Materials and methods}

\section{Zebrafish maintenance}

Adult zebrafish (Tupfel long-fin (TL) strain), nr3c1 knockouts (GRKO) and nr3c2 knockouts (MRKO) (Faught \& Vijayan 2018b) were maintained in $10 \mathrm{~L}$ tanks on a recirculating system with a 14:10 light:darkness cycle (Tecniplast, Buguggiate, Italy) according to established animal care protocols approved by the University of Calgary Animal Care Committee (\#AC17-0079) in accordance with the Canadian Council for Animal Care. Water was maintained at $28.5^{\circ} \mathrm{C}, \mathrm{pH} 7.6$, and $750 \mu \mathrm{S}$ conductivity. Animals were fed twice daily with Gemma micro 300 diet (Skretting, Tooele, UT, USA) in the morning and live Artemia (San Francisco Bay Brand, Newark, CA, USA) in the afternoon.

\section{Cortisol treatment and larval rearing}

Fish larvae were reared for days $0-5$ days post fertilization (dpf) in a $28.5^{\circ} \mathrm{C}$ incubator in $20 \times 100 \mathrm{~mm}$ dishes with $1 \times$ embryo media. Day 3 larvae were transferred to a six-well plate, each well contained 20 larvae and $4 \mathrm{~mL}$ of embryo media. Larvae were exposed to either a vehicle $(0.05 \%$ ethanol) or cortisol (hydrocortisone; Sigma; $5 \mu \mathrm{g} / \mathrm{mL}$ )). At $5 \mathrm{dpf}$, larvae were transferred to $3 \mathrm{~L}$ tanks with $1.5 \mathrm{~L}$ of water. Larvae were fed Gemma micro 150 twice daily. 50\% of the water, dosed appropriately, was exchanged daily until 15 dpf. 15 dpf larvae were euthanized with MS-222 (0.4 mg/L, Sigma). Each $n$ represents a pool of 3-5 larvae. After sampling, larvae were rinsed with $1 \times$ PBS and stored at $-80^{\circ} \mathrm{C}$ for later analysis.

\section{Protein quantification}

Protein quantification was done by homogenizing samples in $50 \mathrm{mM}$ Tris buffer ( $\mathrm{pH}$ 7.5) with a protease inhibitor cocktail (Roche, 04693116001). The homogenate was sonicated (3 s pulse, $\sim 5 \times)$ and centrifuged $(13,000 \boldsymbol{g}$ for $2 \mathrm{~min})$. The supernatant was removed, and the protein concentration was determined using the bicinchoninic acid (BCA) method using bovine serum albumin (BSA) as the standard. The homogenate was then diluted with Laemmli's buffer (156.25 mM Tris, 50\% glycerol, 5\% SDS, $0.0625 \%$ bromophenol blue and 25\% 2-mercaptoethanol). Samples were stored at $-20^{\circ} \mathrm{C}$. Equal amounts of protein (40 $\mu \mathrm{g}$ ) were dotted onto a nitrocellulose membrane and air dried for $2 \mathrm{~h}$. Membranes were then blocked with a solution of powdered skim milk (5\% w/v in TTBS (20 mM Tris, $300 \mathrm{mM} \mathrm{NaCl}, \mathrm{pH} 7.5$ with Tween 0.1\%) containing 0.02\% sodium azide). Primary antibody: antihuman insulin (Sigma) used at a dilution of 1:1000. Antiinsulin was prepared in 5\% BSA + 0.02\% sodium azide and membranes were incubated overnight at $4^{\circ} \mathrm{C}$. Membranes were then washed with TTBS (5 min, $3 \times)$ and incubated for $1 \mathrm{~h}$ with secondary antibody (1:3000 goat anti-rabbit IgG; Bio-Rad, 170-6515). The dots were detected with Clarity Western ECL substrate (BioRad, 170-5061).

\section{Transcript abundance}

Transcript levels of specific genes were measured by quantitative real-time PCR (qPCR). Total RNA was extracted from larvae using Ribozol reagent (VWR, Edmonton, Alberta, Canada) according to the manufacturer's instructions and quantified using a SpectraDrop MicroVolume microplate (VersaMax, Molecular Devices). One microgram of RNA was treated with DNase I (Thermo Scientific) to remove genomic contamination prior to cDNA synthesis using the High-Capacity cDNA RT Kit (Applied Biosystems) according to the manufacturer's protocols. Transcript levels were measured by qPCR in duplicate using gene-specific primers (see Table 1) as described previously (Faught \& Vijayan 2018a).

\section{Statistics}

Data are shown as mean \pm S.E.M. and statistical comparison analyzed using Sigma Plot 13 (Systat Software, San Jose, CA, USA). All data were analyzed using a one-way ANOVA (Holm-Sidak post hoc). Gene transcript abundance and cortisol levels were transformed to meet the assumptions of normality and equal variance. Untransformed data are shown in all figures. Significance level (a) of $P<0.05$ was used in all cases.

\section{Results}

\section{Growth regulation by $M R$ and $G R$ signaling}

Using MRKO and GRKO zebrafish larvae, combined with exogenous cortisol treatment, we established a CR activation paradigm based on the readout of GR- and MR-responsive gene expression (Faught \& Vijayan 2019b). Briefly, CR activation (Fig. 1A) is represented as:

i) None: MRKO larvae only have GR, but because they have basal cortisol levels there is no GR activation.

ii) MR: WT larvae have both $\mathrm{MR}$ and GR, but GR is inactive under basal cortisol levels. 
Table 1 Gene-specific primers.

\begin{tabular}{|c|c|c|}
\hline Gene & Protein product & Forward $\left(5^{\prime}-3^{\prime}\right)$ \\
\hline lepr & Leptin receptor & GGTCTCACTGCCTGTCCATT \\
\hline lepa & Leptin & TCGTCAGAATCAGGGAACAC \\
\hline mstnb & Myostatin & AGACCGCTGTGGCTGCTCAT \\
\hline myog & Myogenin & AGTGGACAGCATAACGGGAACAG \\
\hline igf1 & Igf1 & CCACGATCTCTACGAGCACA \\
\hline ddit4 & Redd1 & ATGCAAGATCAGTTGATTTCCAGCC \\
\hline trim63a & Murf1a & GGAAGAAAACTGCCAGGCACAG \\
\hline trim63b & Murf1b & CAGGACAATGCTCAACGTGCC \\
\hline actb & $\beta$-Actin & GTCCCTGTATGCCTCTGG \\
\hline
\end{tabular}

\begin{tabular}{l} 
Reverse $\left(5^{\prime}-3^{\prime}\right)$ \\
\hline AGATGGTGCTGCTCCACT \\
CCCAATGATGAGCGTTGGA \\
GCGGAAAGCACTGGTAATGT \\
GCTGGTCTGAAGGTAACGGTGAG \\
TCGGCTCGAGTTCTTCTGAT \\
TCAGCATTCTTCAATCAGGAGCTCT \\
CTGGGTGATCTGCTCCAGAAGATG \\
CTTGCTCTTTGCCAATACGCTCTAAGAG \\
AAGTCCAGACGGAGGATG
\end{tabular}

\section{Source}

Liu et al. 2010

Liu et al. 2012

Nesan et al. 2012

Nesan et al. 2012

Nesan et al. 2012

Feng et al. 2012

Shimizu et al. 2017

Shimizu et al. 2017

Nesan \& Vijayan 2016 iii) $M R^{*}$ : GRKO larvae are hypercortisolemic, but lack GR, and, therefore, this group represents MR activation under high cortisol levels.

iv) $M R+G R$ : WT larvae treated with cortisol will activate both GR and MR.

v) GR: MRKO larvae treated with cortisol represents a scenario in which only GR is activated.

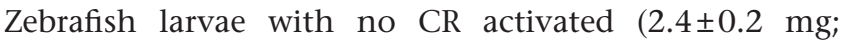
$P=0.350)$ were comparable in weight to MR activated larvae $(2.2 \pm 0.09 \mathrm{mg})$ and $\mathrm{MR}^{*}$ larvae $(2.8 \pm 0.2 \mathrm{mg} ; P=0.142)$ (Fig. 1B). Activation of GR in WT (MR + GR activation) reduced the mass by half $(1.3 \pm 0.1 \mathrm{mg})$ compared to the vehicle-treated WT larvae (MR activation; $P<0.001$ ).
In fish lacking MR (GR activation; $2.9 \pm 0.4 \mathrm{mg}$ ), this effect of cortisol was abolished and the larvae were similar in size to MR activated under high cortisol ( $\mathrm{MR}^{*}$ activation; $P=0.512)$ and no CR activation $(P=0.522)$, but larger than MR activation under basal cortisol conditions $(P=0.010)$. Total larval protein content showed a clear response to MR or GR activation (Fig. 1C). Fish lacking CR activation had $30.8 \pm 1.0 \mathrm{mg}$ total protein/larva. When MR was activated under low cortisol conditions (WT), total protein content increased $(48.7 \pm 0.2 \mathrm{mg} / \mathrm{larva}, P<0.001)$, and this increase was even greater when MR was activated under high cortisol levels (MR*; $64.0 \pm 6.06 \mathrm{mg} / \mathrm{larva}$ ) compared to no CR activation (Fig. 1C). Activation of GR in the presence of MR caused a significant loss of protein $(21.1 \pm 0.3 \mathrm{mg})$
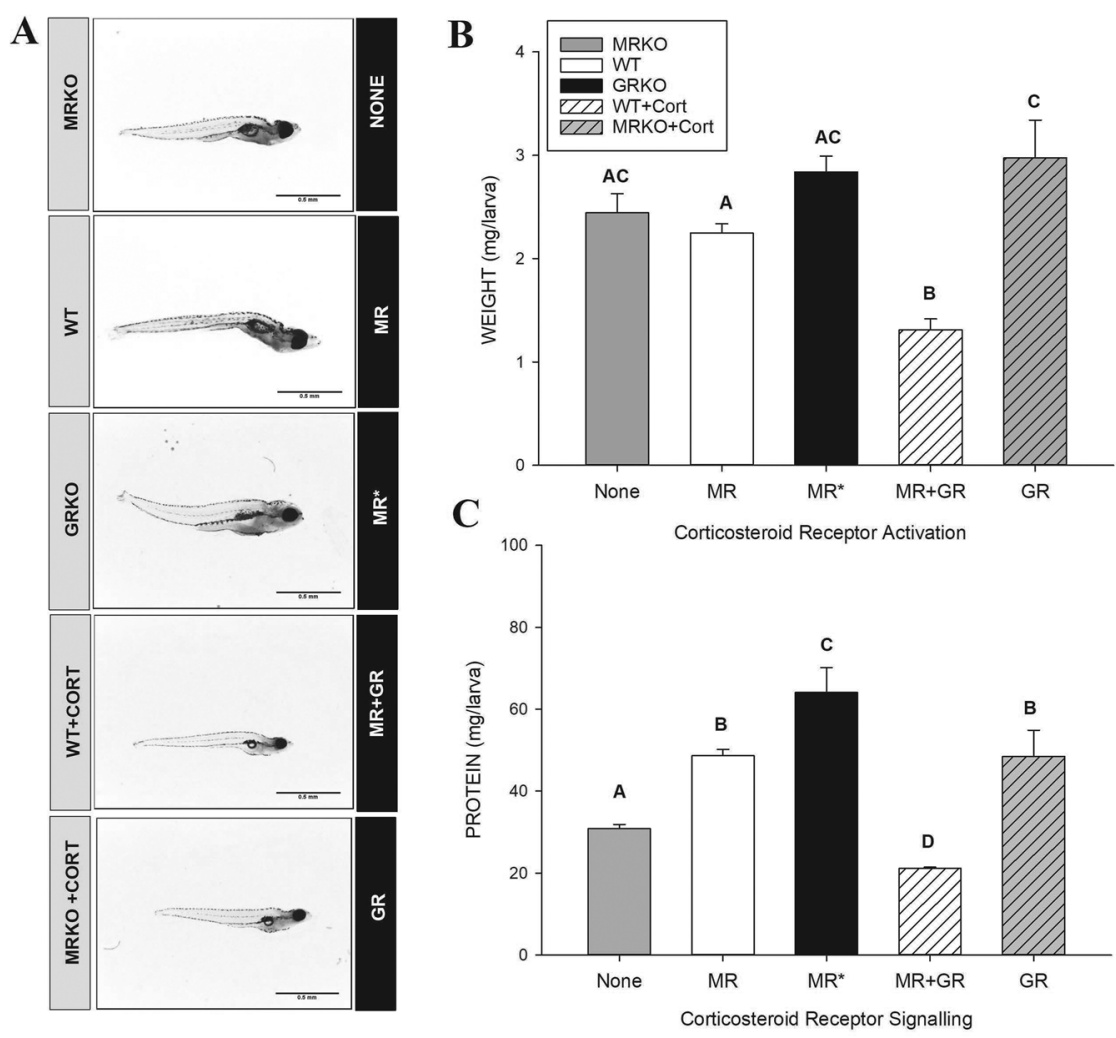

\section{Figure 1}

Regulation of larvae growth by MR and GR signaling. (A) Representative zebrafish larvae at 15 dpf which represent different $C R$ activation. MRKO larvae have no CR activated, WT larvae have only MR activated, GRKO larvae have MR activated under high cortisol conditions (MR*), WT larvae treated with cortisol $(5 \mu \mathrm{g} / \mathrm{mL})$ have both MR and GR activated, and MRKO larvae treated with cortisol only have GR activated. Scale bar length is $0.5 \mathrm{~mm}$. (B) Weight of larvae $(n=10-20)$, and (C) protein content $(n=10-20)$. All bars are \pm S.E.M., $P<0.05$, statistical differences were evaluated with a one-way ANOVA. Bars with different letters denote significance. None (no $C R$ activation); MR (Wildtype); MR* (GRKO); MR + GR (Wildtype + cortisol); GR (MRKO + cortisol). 
compared to all other groups. This cortisol-mediated protein breakdown was abolished in the GR group in the absence of MR (GR; $48.5 \pm 6.4 \mathrm{mg}$ ), which had protein content similar to that of the MR activated (WT) larvae $(P=0.920$; Fig. 1C).

\section{GR activation stimulates proteolysis}

To determine the extent to which cortisol-stimulation of proteolysis were involved in the differences observed in body mass and protein content, the transcript abundance of redd1, murf1 $a$ and murf $1 b$ were quantified. MR activation, under high cortisol levels $\left(\mathrm{MR}^{*}\right)$, decreased redd1 transcript abundance compared to the MR activated (WT) larvae $(P=0.019)$. Cortisol treatment stimulated redd1 transcript abundance seven-fold in both WT $(\mathrm{MR}+\mathrm{GR} ; P<0.001)$ and only GR-activated larvae (MRKO + cortisol; $P<0.001)$ (Fig. 2A). Similarly, exogenous cortisol treatment increased mRNA levels of murf1a fourfold in the MR+GR-activated larvae, and seven-fold in the GR-activated larvae compared to the MR-activated larvae $(P<0.001)$ (Fig. $2 \mathrm{~B})$. MR activated larvae had similar murf1a transcript abundance compared to larvae with no $\mathrm{CR}$ activation ( $\mathrm{MR}^{*}, P=0.515$; MR, $P=0.645$ ). Also, murf $1 b$ transcript abundance RNA levels were significantly higher in GR activated larvae (WT+cortisol; $\mathrm{MRKO}+$ cortisol) compared to the WT (MR activation; $P<0.001$ ) (Fig. 2C). MR activation under high cortisol level had lower murf1b transcript abundance compared to MR activated larvae with basal cortisol levels ( $P=0.004$; Fig. 2C).

\section{Myogenin (myog) but not myostatin (mystnb) is regulated by GR}

There was no effect of genotype or cortisol treatment on the transcript abundance of mystnb $(P=0.410$; Fig. 3A). MR activation did not significantly affect myog transcript abundance $(P<0.001)$ compared to no CR activation (none; Fig. 3B). GR activation either with MR (MR+GR activation) or in the absence of MR (GR activation) significantly reduced myog transcript abundance compared to the $\mathrm{MR}^{*}$ activation larvae $(P<0.001$; Fig. $3 \mathrm{~B})$. There was no difference in myog transcript levels between MR activation larvae and MR+GR activation larvae ( $P=0.134$; Fig. 3B).

\section{MR and GR regulate GH/insulin/IGF1 expression}

To determine the endocrine factors involved in the anabolic processes, we examined the transcript abundance of GH and IGF1, as well as the protein expression of insulin (Fig. 4). The transcript abundance of $g h$ was lower in MR activated fish compared to larvae with no CR activation (Fig. 4A). GR activation in the presence of MR (MR + GR activation) increased $g h$ transcript abundance nearly three-fold in comparison to MR activated larvae $(P<0.001)$ and larvae with no CR activation (Fig. 4A). Activation of GR alone, without the presence of MR, lowered $g h$ transcript abundance to levels similar to larvae with no CR activation $(P=0.133)$, but not different from the MR+GR-activated larvae $(P=0.292$; Fig. $4 \mathrm{~A})$.

There were no differences in igf 1 transcript abundance between larvae with no CR activation and larvae in which
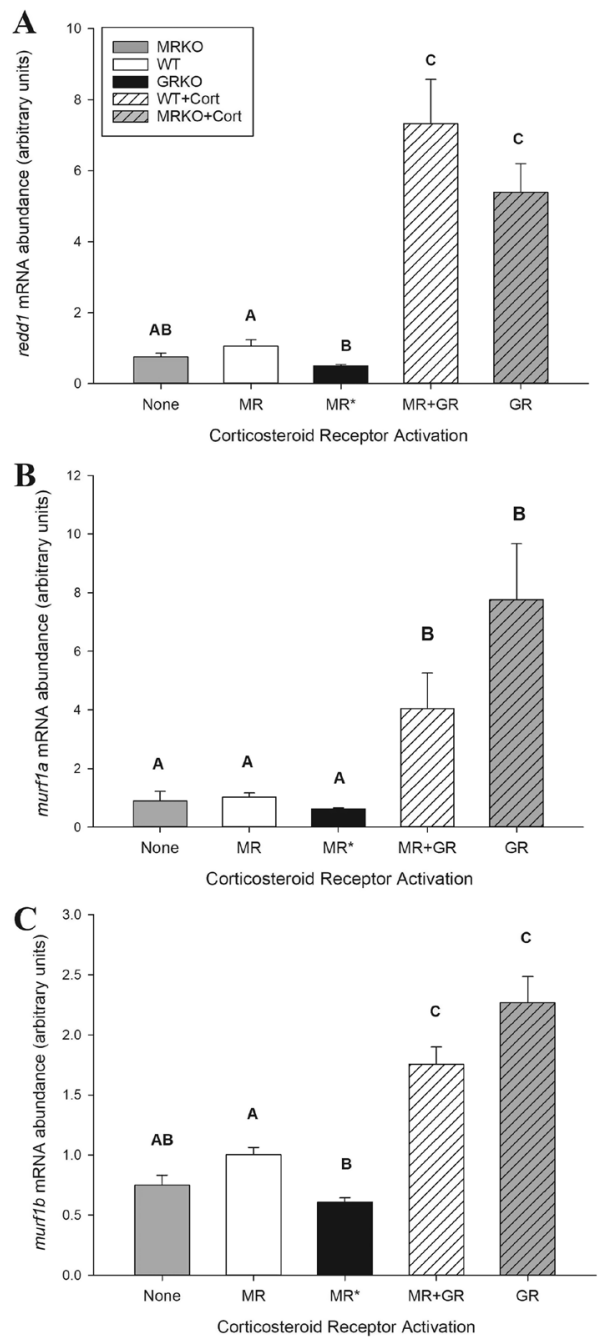

Figure 2

GR activation stimulates proteolysis. Transcript abundance of proteolytic genes involved in skeletal muscle atrophy in zebrafish larvae (15 dpf) treated with cortisol. Transcript abundance of the (A) redd1, (B) murf1a and (C) murf1b. All bars are \pm S.E.M., $P<0.05(n=4-6)$, statistical differences were evaluated with a one-way ANOVA. Bars with different letters denote significance. None (no CR activation); MR (Wildtype); MR* (GRKO); MR + GR (Wildtype + cortisol); GR (MRKO + cortisol). 

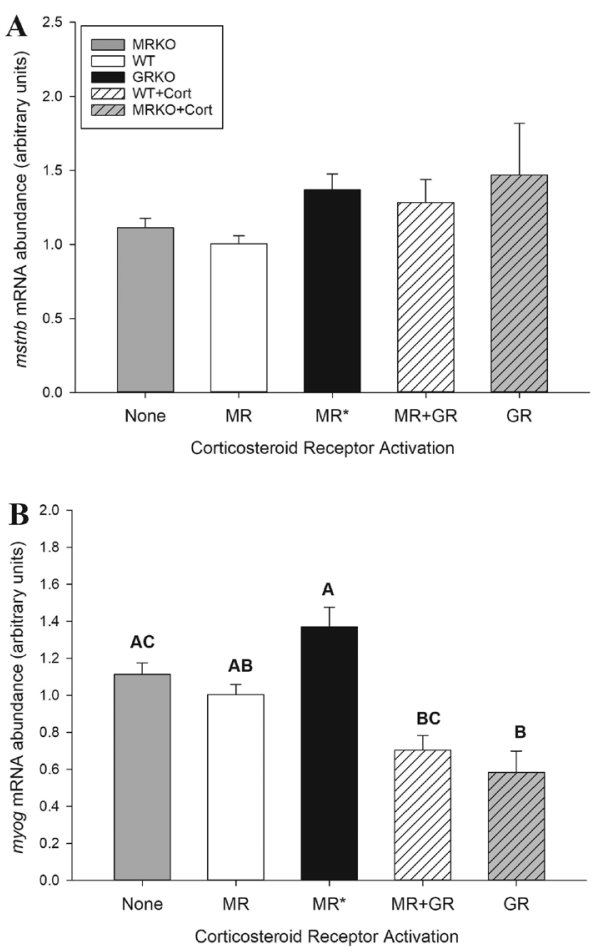

Figure 3

Myogenin (myog) but not myostatin (mystnb) is regulated by GR. (A) Myostatin b (mystnb) and (B) myogenin (myog) gene transcription in $15 \mathrm{dpf}$ zebrafish. All bars are \pm S.E.M., $P<0.05(n=4-6)$, statistical differences were evaluated with a one-way ANOVA. Bars with different letters denote significance. None (no CR activation); MR (Wildtype); MR* (GRKO); MR + GR (Wildtype + cortisol); GR (MRKO + cortisol).

MR was activated (MR; $P=0.930$ and $\left.\mathrm{MR}^{*} ; P=0.990\right)$. I $g f 1$ transcript abundance is higher when GR is activated in the presence of MR (MR+GR; $P=0.016)$, but not when GR alone is activated (Fig. 4B). GR activation without MR returns igf1 transcript abundance to levels seen in larvae that were no treated with cortisol (none, MR and MR* larvae; Fig. 4B).

Insulin expression was similar between larvae with no CR activation and MR activation under low cortisol levels (none vs MR, $P=0.230$; Fig. $4 \mathrm{C}$ ). Activation of MR under high cortisol levels $\left(\mathrm{MR}^{*}\right)$, and in the presence of GR activation (MR+GR larvae) resulted in greater insulin expression compared to the MR activation (WT) larvae ( $P=0.016$ and $P=0.009$, respectively; Fig. $4 \mathrm{C})$. GR activation, in the absence of MR, did not increase insulin expression and was similar to larvae with no CR activation $(P=0.937)$ or MR activation $(P=0.100)$ (Fig. 4C).

\section{Regulation of leptin and leptin receptor by GR and MR}

Activation of MR increased lepa mRNA ten-fold compared to larvae that did not have MR activation (Fig. 5C).
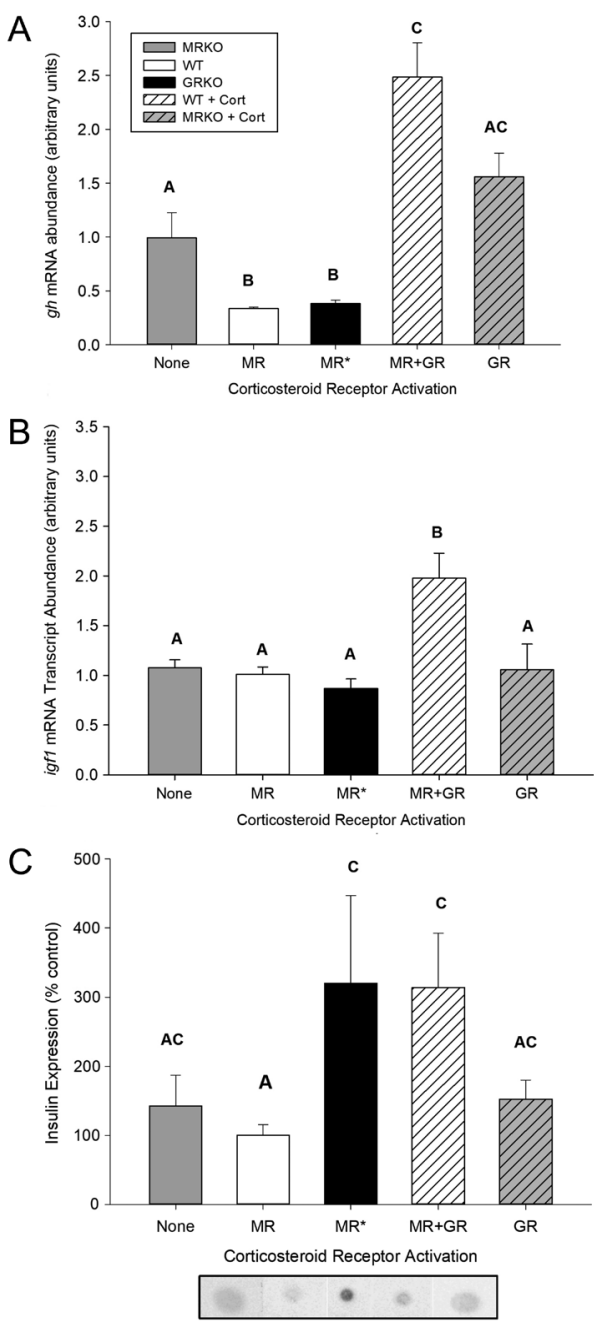

Figure 4

MR and GR regulate GH/insulin/IGF1 expression. To determine somatotropic axis potential transcript abundance of (A) growth hormone (gh) and (B) insulin-like growth factor 1 (igf1) transcript abundance was determined in $15 \mathrm{dpf}$ zebrafish treated with cortisol for 12 days (3-15 dpf). (C) Insulin, as a key player in anabolic processes, was also measured. Insulin expression is represented as \% control (vehicle-treated WT) and a representative dot blot is show below the graph. All bars are \pm S.E.M. $(n=4-6)$, one-way ANOVA was used to determine significance, $P<0.05$. Bars with different letters denote significance. None (no CR activation); MR (Wildtype); MR* (GRKO); MR + GR (Wildtype + cortisol); GR (MRKO + cortisol).

GR activation with exogenous cortisol treatment had no effect on lepa compared to MR-activated larvae (WT, $P=0.42$ ). MR activation under high cortisol levels $\left(\mathrm{MR}^{*}\right)$ decreased the lepa transcript abundance by half compared to the MR-activated larvae $(P=0.014)$ or the MR+GR-activated larvae $(P=0.026$; Fig. $5 \mathrm{~A})$.

MR activation reduced lepr transcript abundance in all cases (Fig. 5B). Larvae with no CR activation had three-fold higher lepr transcript abundance compared to larvae where MR was active. GR activation in the absence 

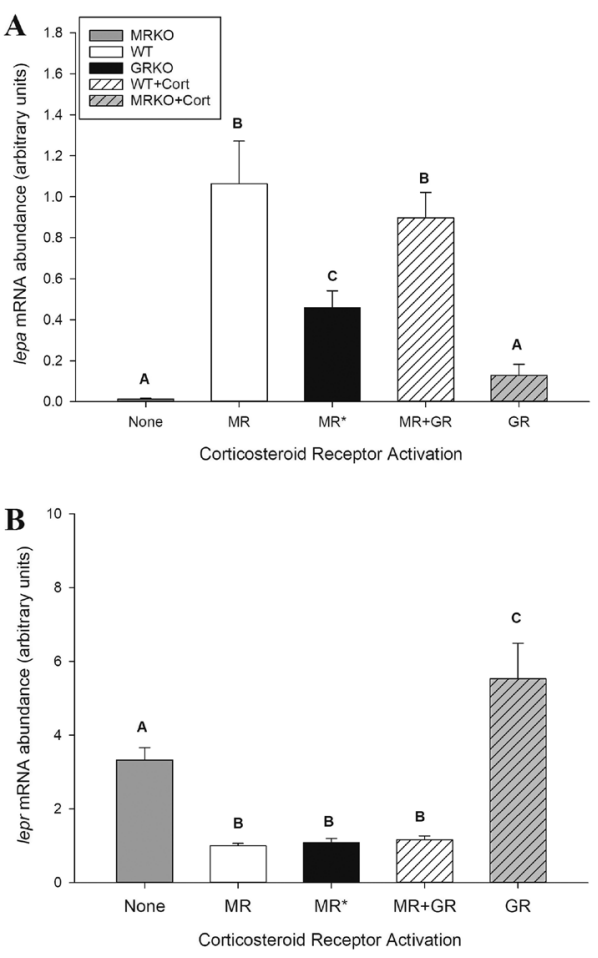

\section{Figure 5}

Regulation of leptin and leptin receptor by GR and MR. Transcript abundance of (A) leptin A (lepa) and (B) leptin receptor (lepr) were assessed. All bars are \pm S.E.M. $(n=4-6)$, a one-way ANOVA was used to determine the significance, $P<0.05$. Bars with different letters are significantly different. None (no CR activation); MR (Wildtype); MR* (GRKO); MR + GR (Wildtype + cortisol); GR (MRKO + cortisol).

of MR increased lepr transcript levels six-fold compared to MR-activated larvae, and two-fold compared to larvae with no $C R$ activation $(P=0.045$; Fig. 5B).

\section{Discussion}

We report an important role for MR activation in the regulation of postnatal protein content in response to GC stimulation in zebrafish larvae. The negative effect of excess GC on growth is conserved in vertebrates. From chronically stressed salmonid parr (McCormick et al. 1998) to steroid treatment in children (Kelly et al. 2012), excess GCs during the postnatal periods are associated with reduced growth (Cianfarani et al. 2002, Greenwood $\&$ Bell 2003), but the mechanisms are unclear. Our results indicate that excess cortisol-mediated growth suppression during postnatal development requires the activation of both MR and GR in zebrafish. MR activation by itself, in the absence of GR activation, led to protein accumulation indicating an anabolic role for this receptor activation. Paradoxically, both MR and GR are required for cortisolmediated protein breakdown, as larvae with GR activation alone had similar larval protein content compared to WT larvae (MR activation). This is the first study to show that both GR and MR activation cooperatively mediates the cortisol-mediated growth suppression during postnatal development in any animal model.

Excess cortisol promotes proteolysis of skeletal muscle mass in mammals, and this is due to the upregulation of several ubiquitin ligases, and regulators of mTOR, which are GR responsive (Kuo et al. 2013, Britto et al. 2014). This also is the case in zebrafish larvae, as GR activation upregulates murf1a, murf1b, and redd 1 transcript levels, supporting a conserved role for GCs in muscle proteolysis (Faught \& Vijayan 2019a). In all cases of the measured muscle proteolytic genes (Fig. 2), GR and not MR is the primary transcription factor regulating these genes. Also, myog, a transcription factor involved in skeletal muscle development and protein synthesis (Kuo et al. 2013), was downregulated in response to GR activation compared to larvae in which only MR was activated under high cortisol levels (Fig. 3B). These results support the conserved role of GR activation as a key mediator of muscle wasting and negative protein balance in response to excess GCs during chronic stress (Kuo et al. 2013). Neither cortisol treatment nor genotype had an effect on mystnb; however, the role of mystnb in teleost is thought to be divergent from its role in mammals (Galt et al. 2014).

Our results indicate that MR by itself has a minimal role in cortisol-mediated proteolysis. This may have evolutionary significance, as $\mathrm{MR}$ is considered to be the type I CR, mediating actions under basal cortisol conditions, while GR, the type II CR, is primarily responsible for mediating GCs action post-stress (Joëls \& de Kloet 2017). The chronic stress-mediated muscle protein breakdown occurring during periods of elevated cortisol levels will increase available amino acids for hepatic gluconeogenesis (Mommsen et al. 1999). The increase in transcript abundance of proteolytic genes in zebrafish supports a conserved role for GR activation in promoting muscle wasting (Faught \& Vijayan 2019a). While the role of MR signaling in protein metabolism has received little attention, our results suggest that MR activation by basal cortisol levels during early development may be essential for postnatal growth promotion. This is evident by the increased larval protein content in response to MR activation in the zebrafish larvae. However, the role of MR may be more complex as the protein breakdown seen with high cortisol also requires the activation of MR in zebrafish larvae. The MRKO group treated with exogenous cortisol (GR only activation) did not show a decrease in larval protein content, confirming that 
MR-GR interaction is required for cortisol-mediated protein breakdown in zebrafish. Given the ancient origin of these two receptors in vertebrates (Baker et al. 2013), we propose that the balance between GR and MR activation may be a key factor in the metabolic adjustments under both basal and stressed conditions. The mechanisms of interaction between these two receptors remains to be determined.

It is generally accepted that cortisol signaling, especially in terms of metabolic regulation, occurs through GR in fish (Mommsen et al. 1999, Mommsen 2001). There is evidence that MR is involved in lipid accumulation and adipogenesis in mammals (Marzolla et al. 2012), and this was also recently shown in zebrafish (Faught \& Vijayan $2019 b$ ). Furthermore, activation of MR in human skeletal muscles with the GR agonist prednisolone regulated a suite of genes similar to that seen in response to GR activation by the same agonist (Chadwick et al. 2015). This suggests that in a metabolic capacity there may be a strong interaction between GR and MR signaling, but the extent to which MR regulate key metabolic genes, either alone or in conjunction with GR in response to cortisol stimulation, is unknown. The capacity for increased protein content in the MR-activated larvae is supported by the lower expression of redd 1 and murf $1 b$ in the MR* groups (Fig. 2A and C), suggesting also an anti-catabolic role for $\mathrm{MR}$ in promoting protein deposition. Under basal conditions, $\sim 80 \%$ of MR are bound by GCs (de Kloet \& Reul 1987), but there is little interaction of MR with GREs as indicated by the absence of an activation of GR-responsive gene (Fig. 2). MR interaction with GREs occurs under primarily under high cortisol levels (Mifsud $\&$ Reul 2016), and this may be the reason for the reduction in transcript changes seen in the redd 1 and murf $1 b$ in the $\mathrm{MR}^{*}$ groups, which are hypercortisolemic (Ziv et al. 2013, Faught \& Vijayan 2018a, 2019a). This suggests that the role of MR, even under stress conditions, is to limit catabolic processes, and this may be a compensatory mechanism to facilitate metabolic recovery post stress. A recent study in mammals reported a direct role for MR activation in the transcriptional regulation of genes involved in skeletal muscle proteolysis (Chadwick et al. 2017). This does not appear to be the case in larval zebrafish (Fig. 2), suggesting that the MR-induced anabolic processes may not only be due to a direct transcriptional control of muscle protein metabolism, but may also include compensatory mechanisms, such as the stimulation of insulin and growth hormone signaling axis. Interestingly, the MRKO group treated with exogenous cortisol (GR only activation) has similar protein levels to WT (Fig. 1C). While we cannot rule out post-transcriptional or post-translational mechanisms affecting the proteolytic capacity of redd1, and murf1 $a, b$ (Fig. 2), we would also like to propose that the anabolic compensation by MR may only occur under conditions of muscle loss, as in the case of cortisol treated WT. Our results fail to completely explain the lack of protein loss in this GR activated group, but suggests that the interaction of GR and MR may be dependent on other factors, including the nutritional/metabolic status of the animal. While the mechanisms surrounding the interaction of GR and MR is unclear, recent work suggests that either heterodimerization (Kiilerich et al. 2015, Mifsud \& Reul 2016), tethering (Rivers et al. 2019), or steric interference in the promoter regions of target genes may be involved (Mifsud \& Reul 2018).

To test the hypothesis that the effect of MR on protein deposition may involve regulation of anabolic hormones, we measured the expression of insulin, and transcript abundance of GH and IGF1 in zebrafish larvae. To date, the effects of cortisol on GH regulation in fish are associative at best, and the mechanisms involved are far from clear. For instance, during periods of rapid growth, as in the case of Atlantic salmon parr, repeated stress, and the attendant rise in cortisol was associated with an increase in circulating GH levels (McCormick et al. 1998). However, in adult rainbow trout, there was no change in GH levels with acute stress (Shepherd et al. 2011), which suggests that GH stimulation during stress may be related to the developmental stage, and maturity of the animal. Also, in chronically stressed rainbow trout higher transcript abundance of the two GH paralogs was associated with lower circulating GH levels, but increased hepatic igf1 mRNA (Madison et al. 2015). In these studies, it was unknown whether GR, MR or interaction of these receptors were involved in mediating the cortisol-induced changes in GH levels. Here we show that activation of both GR and MR regulates the $g h / i g f 1$ transcript abundance during early development in zebrafish. The transcript abundances of $g h$ and igf1 are increased when both GR and MR are activated (MR+GR larvae), but neither GR nor MR activation alone affects these genes (Fig. 4). This lack of regulation of $g h$ and igf 1 transcripts with MR activation alone suggests that these anabolic hormones may not be the MR targets promoting the protein accumulation observed in these larvae. However, during chronic stress and high cortisol levels, MR increases insulin expression, and this may promote protein synthesis. This notion is supported by the recent observation that GRKO adult zebrafish, in which MR is activated under high cortisol levels, showed a higher glucose uptake by the 
skeletal muscle, and this was associated with increased phosphorylation of translation elongation factor eIF4B and protein synthesis (Faught \& Vijayan 2019a).

Further support for better glucose metabolism in response to MR activation is seen by the changes in lepa and lepr transcript abundance. The role of leptin in the control of feeding in the lower vertebrates is not as clear as in mammals (Michel et al. 2016). For instance, leptin expression is more pronounced in the liver than in the adipose tissue and regulates glucose levels in teleosts (Michel et al. 2016). Stress and the attendant rise in GCs modulate leptin expression in mammals (Zakrzewska et al. 1997) and fishes (Löhr et al. 2018); however, the effect of GCs on circulating leptin levels lacks consensus. In humans, prolonged treatment with GCs increases circulating levels of leptin and inhibits food intake (Uddén et al. 2003), but reducing cortisol synthesis has also been shown to increase serum leptin concentrations in humans (Copeland et al. 2011). Leptin can also attenuate corticosterone-induced increases in plasma insulin levels (Solano \& Jacobson 1999), suggesting that GCs/leptin interaction may be important in maintaining energy substrate homeostasis. GCs will counteract the effects of leptin, as exogenous GC treatment will reverse leptin-induced reductions in weight gain and body fat in mammals (Solano \& Jacobson 1999). Interestingly, we are showing for the first time that MR activation may be important in maintaining transcript abundance of lepa, the primary paralog of leptin in zebrafish (Michel et al. 2016). However, MR activation also reduced the lepr transcript abundance leading to the proposal that MR activation may cause leptin resistance in zebrafish. The higher insulin expression in response to MR activation in the presence of high cortisol supports leptin resistance, as lepr-knockout zebrafish had higher beta-cell number and hyperinsulinemia (Michel et al. 2016). Further work needs to be carried out to fully elucidate the interaction between GCs and leptin signaling in facilitating the energy repartitioning during postnatal growth in zebrafish. Taken together, our results suggest that the promotion of protein deposition by MR under elevated cortisol levels (MR* activation) may involve a suppression of proteolytic genes, coupled with an increase in insulin responsiveness, but further studies are warranted to elucidate the mechanisms involved. These results suggest that the presence of both GR/MR plays an essential role in maintaining the balance between the anabolic and catabolic pathways during stress. Consequently, dysregulation of these pathways by differentially activating MR or GR may lead to marked differences in growth in zebrafish in response to GC stimulation.
In conclusion, MR and GR activation plays a key physiological role in the regulation of postnatal growth in zebrafish. While adaptive in the context of starvation or acute infection, prolonged periods of elevated GCs during early development can result in muscle loss and long-term developmental dysfunction. Here we show that MR activation, under both basal and stressed conditions are critical for the regulation of protein status (Fig. 6). MR activation stimulates protein deposition, and this includes inhibiting proteolytic genes (anti-catabolic) and stimulating insulin responsiveness (anabolic processes). GR is activated under high cortisol levels and will stimulate proteolysis, but the protein breakdown seen with excess cortisol requires the activation of both GR and MR (Fig. 6). We propose that MR may be playing a compensatory role in protein recovery post-stress and this requires the interaction with GR and involves upregulation of the gh/igf1/insulin signalling. MR modulation of leptin resistance may also be another mechanism involved in energy repartitioning during cortisol stimulation. A more detailed study in WT fish involving the manipulation of cortisol levels, including complete inhibition of cortisol synthesis may help to elucidate the physiological and evolutionary significance of chronic MR signaling. Overall, both MR and GR activation have key roles to play in protein homeostasis in response to stress during

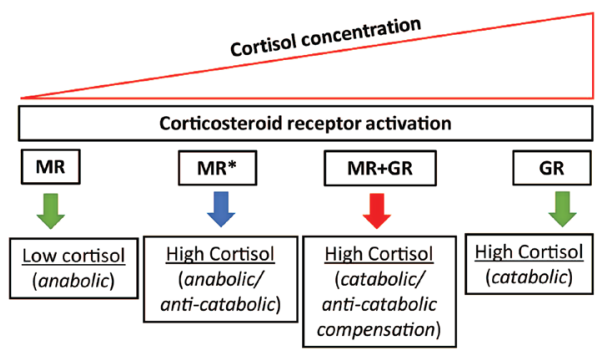

\section{Figure 6}

A working model of $\mathrm{CR}$ activation on postnatal growth. A schematic diagram of how GR and MR activation modulates growth processes. Under basal cortisol conditions only MR is active, and this promotes anabolic processes such as protein deposition. As the cortisol concentration increases, MR signaling is amplified as seen with increased protein, insulin levels and a marked decrease in key proteolytic genes (combination of anabolic and anti-catabolic processes). However, the activation of GR alone, strongly upregulates catabolic processes such as transcription of proteolytic genes (redd1, murf1a and murf1b). Our results indicate that activation of both MR and GR is required for protein breakdown, while the increase in gh/igf1 abundance suggests anticatabolic compensation perhaps mediated by MR. Several caveats to this working hypothesis remain to be determined including (i) whether lack of GR amplifies MR activity under high cortisol levels (GRKO larvae), (ii) whether the compensatory gh/igf1 response is due to direct stimulation of transcription by MR/GR or indirectly due to changes in the protein/ metabolic status and (iii) whether MR acts post-transcriptionally to mediate proteolysis, as GR activation alone did not reduce protein levels. 
postnatal development. Understanding the mechanisms of these receptor interactions, and the target genes involved may have biomedical applications by reducing and managing stress-related growth dysfunctions. Also, pharmacological intervention of MR signaling may be a possible therapeutic means to reduce muscle wasting associated with excess early life stage GCs treatment.

\section{Declaration of interest}

The authors declare that there is no conflict of interest that could be perceived as prejudicing the impartiality of the research reported.

\section{Funding}

This work was supported by an NSERC discovery grant to M M V (RGPIN \# 217481-2013). E F was supported by an NSERC-PGS doctoral scholarship.

\section{References}

Alsop D \& Vijayan MM 2008 Development of the corticosteroid stress axis and receptor expression in zebrafish. American Journal of Physiology: Regulatory, Integrative and Comparative Physiology 294 R711-R719. (https://doi.org/10.1152/ajpregu.00671.2007)

Alsop D \& Vijayan MM 2009 The zebrafish stress axis: molecular fallout from the teleost-specific genome duplication event. General and Comparative Endocrinology 161 62-66. (https://doi.org/10.1016/j. ygcen.2008.09.011)

Baker ME, Funder JW \& Kattoula SR 2013 Evolution of hormone selectivity in glucocorticoid and mineralocorticoid receptors. Journal of Steroid Biochemistry and Molecular Biology 137 57-70. (https://doi. org/10.1016/j.jsbmb.2013.07.009)

Bernier NJ 2006 The corticotropin-releasing factor system as a mediator of the appetite-suppressing effects of stress in fish. General and Comparative Endocrinology 146 45-55. (https://doi.org/10.1016/j. ygcen.2005.11.016)

Britto FA, Begue G, Rossano B, Docquier A, Vernus B, Sar C, Ferry A, Bonnieu A, Ollendorff V \& Favier FB 2014 REDD1 deletion prevents dexamethasone-induced skeletal muscle atrophy. American Journal of Physiology: Endocrinology and Metabolism 307 E983-E993. (https://doi. org/10.1152/ajpendo.00234.2014)

Britto FA, Cortade F, Belloum Y, Blaquière M, Gallot YS, Docquier A, Pagano AF, Jublanc E, Bendridi N, Koechlin-Ramonatxo C, et al. 2018 Glucocorticoid-dependent REDD1 expression reduces muscle metabolism to enable adaptation under energetic stress. BMC Biology 16 65. (https://doi.org/10.1186/s12915-018-0525-4)

Chadwick JA, Hauck JS, Lowe J, Shaw JJ, Guttridge DC, GomezSanchez CE, Gomez-Sanchez EP \& Rafael-Fortney JA 2015 Mineralocorticoid receptors are present in skeletal muscle and represent a potential therapeutic target. FASEB Journal 29 4544-4554. (https://doi.org/10.1096/fj.15-276782)

Chadwick JA, Bhattacharya S, Lowe J, Weisleder N \& Rafael-Fortney JA 2017 Renin-angiotensin-aldosterone system inhibitors improve membrane stability and change gene-expression profiles in dystrophic skeletal muscles. American Journal of Physiology: Cell Physiology $\mathbf{3 1 2}$ C155-C168. (https://doi.org/10.1152/ajpcell.00269.2016)

Charmandari E, Tsigos C \& Chrousos G 2005 Endocrinology of the stress response. Annual Review of Physiology 67 259-284. (https://doi. org/10.1146/annurev.physiol.67.040403.120816)

(c) 2020 Society for Endocrinology Published by Bioscientifica Ltd. Printed in Great Britain
Cianfarani S, Geremia C, Scott CD \& Germani D 2002 Growth, IGF system, and cortisol in children with intrauterine growth retardation: is catch-up growth affected by reprogramming of the hypothalamicpituitary-adrenal axis? Pediatric Research 51 94-99. (https://doi. org/10.1203/00006450-200201000-00017)

Copeland DL, Duff RJ, Liu Q, Prokop J \& Londraville RL 2011 Leptin in teleost fishes: an argument for comparative study. Frontiers in Physiology 2 26. (https://doi.org/10.3389/fphys.2011.00026)

de Kloet ER \& Reul JMHM 1987 Feedback action and tonic influence of the corticosteroids on brain function: a concept arising from the heterogeneity of brain receptor systems. Psychoneuroendocrinology $\mathbf{1 2}$ 83-105. (https://doi.org/10.1016/0306-4530(87)90040-0)

Facchinello N, Skobo T, Meneghetti G, Colletti E, Dinarello A, Tiso N, Costa R, Gioacchini G, Carnevali O, Argenton F, et al. 2017 nr3c1 null mutant zebrafish are viable and reveal DNA-binding-independent activities of the glucocorticoid receptor. Scientific Reports $\mathbf{7} 4371$. (https://doi.org/10.1038/s41598-017-04535-6)

Faught E \& Vijayan MM 2018a The mineralocorticoid receptor is essential for stress axis regulation in zebrafish larvae. Scientific Reports $\mathbf{8} 18081$. (https://doi.org/10.1038/s41598-018-36681-w)

Faught E \& Vijayan MM 2018b Maternal stress and fish reproduction: the role of cortisol revisited. Fish and Fisheries 19 1016-1030. (https://doi. org/10.1111/faf.12309)

Faught E \& Vijayan MM 2019a Loss of the glucocorticoid receptor in zebrafish improves muscle glucose availability and increases growth. American Journal of Physiology: Endocrinology and Metabolism 316 E1093-E1104. (https://doi.org/10.1152/ajpendo.00045.2019)

Faught E \& Vijayan MM 2019b Postnatal triglyceride accumulation is regulated by the mineralocorticoid receptor under basal and stress conditions. Journal of Physiology 597 4927-4941. (https://doi. org/10.1113/JP278088)

Feng Q, Zou X, Lu L, Li Y, Liu Y, Zhou J \& Duan C 2012 The stressresponse gene redd 1 regulates dorsoventral patterning by antagonizing Wnt/b-catenin activity in zebrafish. PLoS ONE 7 e52674. (https://doi.org/10.1371/journal.pone.0052674)

Galt NJ, Michael J, Remily EA, Romero SR \& Biga PR 2014 The effects of exogenous cortisol on myostatin transcription in rainbow. Comparative Biochemistry and Physiology, Part A 175 57-63. (https:// doi.org/10.1016/j.cbpa.2014.05.015)

Gokulakrishnan G, Estrada IJ, Sosa HA \& Fiorotto ML 2012 In utero glucocorticoid exposure reduces fetal skeletal muscle mass in rats independent of effects on maternal nutrition. American Journal of Physiology: Regulatory, Integrative and Comparative Physiology 302 R1143-R1152. (https://doi.org/10.1152/ajpregu.00466.2011)

Greenwood PL \& Bell AW 2003 Consequences of intra-uterine growth retardation for postnatal growth, metabolism and pathophysiology. Reproduction 61 195-206. (https://doi.org/10.1530/biosciprocs.5.015)

Joëls M \& de Kloet ER 201730 YEARS OF THE MINERALOCORTICOID RECEPTOR: The brain mineralocorticoid receptor: a saga in three episodes. Journal of Endocrinology 234 T49-T66. (https://doi. org/10.1530/JOE-16-0660)

Kelly HW, Sternberg AL, Lescher R, Fuhlbrigge AL, Williams P, Zeiger RS, Raissy HH, Van Natta ML, Tonascia J, Strunk RC, et al. 2012 Effect of inhaled glucocorticoids in childhood on adult height. New England Journal of Medicine 367 904-912. (https://doi.org/10.1056/ NEJMoa1203229

Kiilerich P, Triqueneaux G, Christensen NM, Trayer V, Terrien X, Lombès M \& Prunet P 2015 Interaction between the trout mineralocorticoid and glucocorticoid receptors in vitro. Journal of Molecular Endocrinology 55 55-68. (https://doi.org/10.1530/JME-150002)

Kuo T, Harris CA \& Wang JC 2013 Metabolic functions of glucocorticoid receptor in skeletal muscle. Molecular and Cellular Endocrinology 380 79-88. (https://doi.org/10.1016/j.mce.2013.03.003)

Lipina C \& Hundal HS 2016 Is REDD1 a metabolic éminence grise? Trends in Endocrinology and Metabolism 27 868-880. (https://doi. org/10.1016/j.tem.2016.08.005) 
Liu Q, Chen Y, Copeland D, Ball H, Duff RJ, Rockich B \& Londraville RL 2010 Expression of leptin receptor gene in developing and adult zebrafish. General and Comparative Endocrinology 166 346-355. (https://doi.org/10.1016/j.ygcen.2009.11.015)

Liu Q, Dalman M, Chen Y, Akhter M, Brahmandam S, Patel Y, Lowe J, Thakkar M, Gregory AV, Phelps D, et al. 2012 Knockdown of leptin A expression dramatically alters zebrafish development. General and Comparative Endocrinology 178 562-572. (https://doi.org/10.1016/j. ygcen.2012.07.011)

Löhr H, Hess S, Pereira MMA, Reinoß P, Leibold S, Schenkel C, Wunderlich CM, Kloppenburg P, Brüning JC \& Hammerschmidt M 2018 Diet-induced growth is regulated via acquired leptin resistance and engages a Pomc-somatostatin-growth hormone circuit. Cell Reports 23 1728-1741. (https://doi.org/10.1016/j.celrep.2018.04.018)

Madison BN, Tavakoli S, Kramer S \& Bernier NJ 2015 Chronic cortisol and the regulation of food intake and the endocrine growth axis in rainbow trout. Journal of Endocrinology 226 103-119. (https://doi. org/10.1530/JOE-15-0186)

Marzolla V, Armani A, Zennaro MC, Cinti F, Mammi C, Fabbri A, Rosano GMC \& Caprio M 2012 The role of the mineralocorticoid receptor in adipocyte biology and fat metabolism. Molecular and Cellular Endocrinology 350 281-288. (https://doi.org/10.1016/j. mce.2011.09.011)

McCormick SD, Shrimpton JM, Carey JB, O'Dea MF, Sloan KE, Moriyama S \& Björnsson BT 1998 Repeated acute stress reduces growth rate of Atlantic salmon parr and alters plasma levels of growth hormone, insulin-like growth factor I and cortisol. Aquaculture 168 221-235. (https://doi.org/10.1016/S0044-8486(98)00351-2)

Michel M, Page-McCaw PS, Chen W \& Cone RD 2016 Leptin signaling regulates glucose homeostasis, but not adipostasis, in the zebrafish. PNAS 113 3084-3089. (https://doi.org/10.1073/pnas.1513212113)

Mifsud KR \& Reul JMHM 2016 Acute stress enhances heterodimerization and binding of corticosteroid receptors at glucocorticoid target genes in the hippocampus. PNAS 113 11336-11341. (https://doi. org/10.1073/pnas.1605246113)

Mifsud KR \& Reul JMHM 2018 Mineralocorticoid and glucocorticoid receptor-mediated control of genomic responses to stress in the brain. Stress 21 389-402. (https://doi.org/10.1080/10253890.2018.1456526)

Mommsen TP 2001 Paradigms of growth in fish. Comparative Biochemistry and Physiology 129 207-219. (https://doi.org/10.1016/S10964959(01)00312-8)

Mommsen TP, Vijayan MM \& Moon TW 1999 Cortisol in teleosts : dynamics, mechanisms of action, and metabolic regulation. Reviews in Fish Biology and Fisheries 9 211-268. (https://doi. org/10.1023/A:1008924418720)

Nesan D \& Vijayan MM 2016 Maternal cortisol mediates hypothalamuspituitary-interrenal axis development in zebrafish. Scientific Reports 6 22582. (https://doi.org/10.1038/srep22582)

Nesan D, Kamkar M, Burrows J, Scott IC, Marsden M \& Vijayan MM 2012 Glucocorticoid receptor signaling is essential for mesoderm formation and muscle development in zebrafish. Endocrinology 153 1288-1300. (https://doi.org/10.1210/en.2011-1559)

Ortega VA, Lovejoy DA \& Bernier NJ 2013 Appetite-suppressing effects and interactions of centrally administered corticotropin-releasing factor, urotensin I and serotonin in rainbow trout (Oncorhynchus mykiss). Frontiers in Neuroscience 7 196. (https://doi.org/10.3389/ fnins.2013.00196)

Pickering AD 1993 Growth and stress in fish production. Aquaculture 111 51-63. (https://doi.org/10.1016/0044-8486(93)90024-S)

Rivers CA, Rogers MF, Stubbs FE, Conway-Campbell BL, Lightman SL \& Pooley JR 2019 Glucocorticoid receptor-tethered mineralocorticoid receptors increase glucocorticoid-induced transcriptional responses. Endocrinology 160 1044-1056. (https://doi.org/10.1210/en.201800819)

Sadoul B \& Vijayan MM 2016 Stress and growth. In Fish Physiology, pp 167-205. Eds CB Schreck, L Tort, AP Farrell \& CJ Brauner. Amsterdam, Netherlands: Elsevier Inc. (https://doi.org/10.1016/B978-0-12-8027288.00005-9)

Sartin JL, Kemppainen RJ, Coleman ES, Steele B \& Williams JC 1994 Cortisol inhibition of growth hormone-releasing hormone-stimulated growth hormone release from cultured sheep pituitary cells. Journal of Endocrinology 141 517-525. (https://doi.org/10.1677/joe.0.1410517)

Shapiro LE, Samuels HH \& Yaffe BM 1978 Thyroid and glucocorticoid hormones synergistically control growth hormone mRNA in cultured GH1 cells. PNAS 75 45-49. (https://doi.org/10.1073/pnas.75.1.45)

Shepherd BS, Aluru N \& Vijayan MM 2011 Acute handling disturbance modulates plasma insulin-like growth factor binding proteins in rainbow trout (Oncorhynchus mykiss). Domestic Animal Endocrinology 40 129-138. (https://doi.org/10.1016/j. domaniend.2010.09.007)

Shimizu N, Yoshikawa N, Ito N, Maruyama T, Suzuki Y, Takeda SI, Nakae J, Tagata Y, Nishitani S, Takehana K, et al. 2011 Crosstalk between glucocorticoid receptor and nutritional sensor mTOR in skeletal muscle. Cell Metabolism 13 170-182. (https://doi. org/10.1016/j.cmet.2011.01.001)

Shimizu H, Langenbacher AD, Huang J, Wang K, Otto G, Geisler R, Wang Y \& Chen JN 2017 The calcineurin-FoxO-MuRF1 signaling pathway regulates myofibril integrity in cardiomyocytes. eLife 6 1-19. (https://doi.org/10.7554/eLife.27955)

Solano JM \& Jacobson L 1999 Glucocorticoids reverse leptin effects on food intake and body fat in mice without increasing NPY mRNA. American Journal of Physiology: Endocrinology and Metabolism 277 E708-E716. (https://doi.org/10.1016/0031-9384(73)90207-2)

Uddén J, Björntorp P, Arner P, Barkeling B, Meurling L \& Rössner S 2003 Effects of glucocorticoids on leptin levels and eating behaviour in women. Journal of Internal Medicine 253 225-231. (https://doi. org/10.1046/j.1365-2796.2003.01099.x)

Yuen KCJ, Chong LE \& Riddle MC 2013 Influence of glucocorticoids and growth hormone on insulin sensitivity in humans. Diabetic Medicine 30 651-663. (https://doi.org/10.1111/dme.12184)

Zakrzewska KE, Cusin I, Sainsbury A, Rohner-Jeanrenaud F \& Jeanrenaud B 1997 Glucocorticoids as counterregulatory hormones of leptin: toward an understanding of leptin resistance. Diabetes $\mathbf{4 6}$ 717-719. (https://doi.org/10.2337/diab.46.4.717)

Ziv L, Muto A, Schoonheim PJ, Meijsing SH, Strasser D, Ingraham H, Schaaf MJ, Yamamoto KR \& Baier H 2013 An affective disorder in zebrafish with mutation of the glucocorticoid receptor. Molecular Psychiatry 18 681-691. (https://doi.org/10.1038/mp.2012.64)

Received in final form 8 October 2019

Accepted 29 October 2019

Accepted Manuscript published online 29 October 2019 https://joe.bioscientifica.com https://doi.org/10.1530/JOE-19-0358 (c) 2020 Society for Endocrinology Published by Bioscientifica Ltd. Printed in Great Britain 